This item was submitted to Loughborough's Research Repository by the author.

Items in Figshare are protected by copyright, with all rights reserved, unless otherwise indicated.

\title{
A wavelet analysis on digital microstructure in microbumps
}

PLEASE CITE THE PUBLISHED VERSION

http://dx.doi.org/10.1109/ICEPT.2015.7236608

\section{PUBLISHER}

(c) IEEE

\section{VERSION}

AM (Accepted Manuscript)

\section{PUBLISHER STATEMENT}

This work is made available according to the conditions of the Creative Commons Attribution-NonCommercialNoDerivatives 4.0 International (CC BY-NC-ND 4.0) licence. Full details of this licence are available at: https://creativecommons.org/licenses/by-nc-nd/4.0/

\section{LICENCE}

CC BY-NC-ND 4.0

\section{REPOSITORY RECORD}

Huang, Zhiheng, Hao Lv, and Paul P. Conway. 2019. "A Wavelet Analysis on Digital Microstructure in Microbumps". figshare. https://hdl.handle.net/2134/20645. 


\section{A Wavelet Analysis on Digital Microstructure in Microbumps}

\author{
Zhiheng Huang ${ }^{*}$ and Hao Lv \\ School of Physics and Engineering \\ Sun Yat-sen University \\ Guangzhou, China \\ *e-mail: hzh29@mail.sysu.edu.cn
}

\author{
Paul Conway \\ School of Mechanical and Manufacturing Engineering \\ Loughborough University \\ Loughborough, United Kingdom \\ e-mail: p.p.conway@lboro.ac.uk
}

\begin{abstract}
Heterogeneous three-dimensional system integration is the ultimate goal for packaging and integration, where materials are pushed to their physical limits. In this context, the microstructure of packaging materials, which exhibits a multi-scale nature, will be carefully designed and tightly controlled in both manufacturing and in-service conditions to ensure long-term reliability of the electronic products. A multi-level discrete wavelet transform using the haar wavelet is conducted on the dendritic structures, simulated with a phase field model, during solidification in microbumps with different sizes and geometries. The statistical data, e.g. the mean, standard deviation and energy, of the detail coefficients from the wavelet analysis reveal a wealthy of information on the features of the dendritic structure and its evolution during solidification at multiple resolutions. The size and geometry effects on the microstructure formed in the microbumps can thus be quantified by such data. Further studies using techniques such as principle component analysis and Radon transform can be conducted to evaluate the consistence of the result.
\end{abstract}

Keywords-microstructure; dendrite; microbump; wavelet analysis; size and geometry

\section{INTRODUCTION}

With the miniaturization and multi-functionalization of the electronic products, 3-D electronic packaging has been developed as a major solution for high density packaging, where the size of interconnects become smaller and smaller, and reached to a microscale. As a result, the reliability of the microbumps has become a critical issue. In a microbump, the influence of microstructure on its reliability can no longer be underestimated. Therefore, the traditional analysis methods on the reliability, in which the effects resulted from the microstructural heterogeneity were not taken into consideration, may provide inaccurate life-time predictions [1]. As a remedy to improve this situation, the microstructures in the microbumps should be carefully studied in the first place. It has been demonstrated that the sizes and geometries of microbumps can influence the microstructural evolution and consequently the properties of microbumps [2]. In addition, Wu et al. have recently simulated the formation of dendrites in microbumps with different sizes and geometries during a solidification process in an undercooled liquid [3]. The four geometries studied include an hourglass, a rectangle, a symmetrical barrel and an asymmetrical barrel. However, the relationship between the microstructure and the properties of microbumps with different sizes and geometries has not yet been quantified. Using a multi-resolution wavelet analysis approach, this study goes one step further to quantify the characteristics of the digital microstructure formed in the microbumps simulated by $\mathrm{Z}$. Wu [4].

\section{Modeling AND ANALYSis Methodology}

\section{A. Phase Field Microstructural Modeling}

A phase-field model developed by Kim et al. [5] is used in this study to simulate the microstructural evolution during solidification in microbumps of four different geometries. The partial differential equations (PDEs) of the phase field models are solved using a finite element method based general PDE solver COMSOL Multiphysics [6]. Further details on the governing equations, boundary conditions and other model parameters are referred to a previous publication [3].

\section{B. Wavelet Analysis on Digital Microstructure}

The wavelet transform is a multi-resolution analysis method for digital signals [7]. In this study, the microstructural simulated using the phase field model are analyzed using the haar wavelet. The digital microstructure inherently contains discontinuity because of its multiphase nature. Unlike the Fourier transform, the wavelet transform can naturally deal with discontinuity. In addition, the microstructures of materials exhibit a multi-scale nature. The multi-level wavelet transform can examine a digital signal at multiple resolutions. Therefore, the wavelet transform is a good tool to quantify the features of material microstructures. The Wavelet Toolbox (Version 4.14.1) in MATLAB [8] is used in this work to analyze the microstructural data in this study.

\section{RESULTS AND DISCUSSION}

In the wavelet analysis of the microstructural signals, the approximation coefficients at different levels describe the characteristics of the low frequency component of the signal, i.e., the information about the signal at a coarser level. In addition, the detail coefficients provide the fine details of the signal. 


\section{A. Microstructure and Wavelet Analysis at $t=1.25 \times 10^{-7} \mathrm{~S}$}

Fig. 1 shows the solidified microstructure at $t=1.25 \times 10^{-7} \mathrm{~s}$ in the microbumps of four different geometries. The four geometries from left to right are referred to as an hourglass, a rectangle, a symmetric barrel and an asymmetric barrel, respectively. Fig. 2 demonstrates the result from the wavelet analysis using the haar wavelet on the microstructure in the hourglass microbump up to level 3.
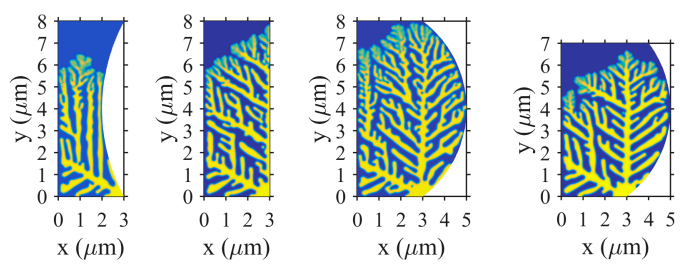

Fig. 1. Microstructure formed in microbumps of four different geometries during solidification at $t=1.25 \times 10^{-7} \mathrm{~s}$.

In a multilevel two-dimensional wavelet analysis, the detail coefficients are given by the horizontal detail coefficient $(\mathrm{H})$, vertical detail coefficient $(\mathrm{V})$ and diagonal detail coefficient (D). The number following the letters ' $\mathrm{H}$ ', ' $\mathrm{V}$ ' and ' $\mathrm{D}$ ' indicates the level of the analysis. In Fig. 2, it can be observed that the diagonal detail coefficient is negligible compared to the horizontal and vertical detail coefficients of the same level. Therefore, it is the horizontal and vertical detail coefficients that are of our primary concern. Noted that the abscissa and ordinate values represent the number of pixels. A resolution of 200 pixels per micron is used throughout this study. It can be further observed that the detail coefficients at level 1 are essentially near zero. It is the detail coefficients at levels 2 and 3 that will be focused on in the following discussion.
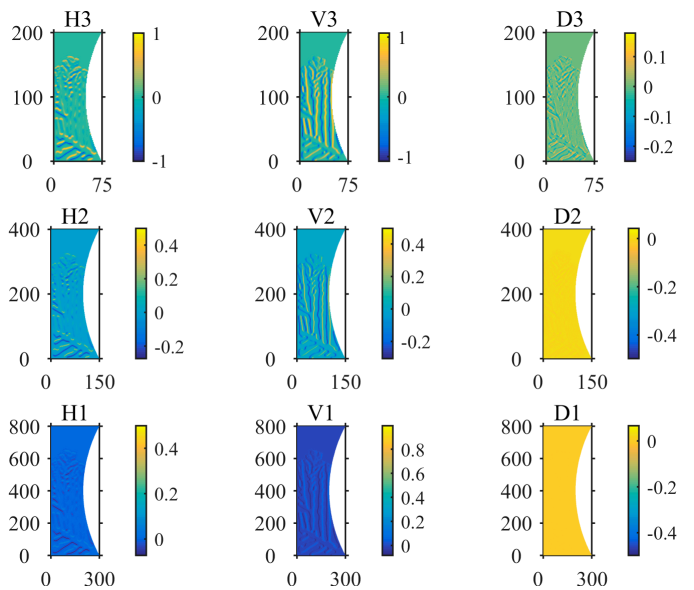

Fig. 2. Three-level wavelet analysis on the microstructure formed in the hourglass during solidifcation at $t=1.25 \times 10^{-7} \mathrm{~s}$.

\section{B. Microstructural and Wavelt Analysis at Four Times}

The microstructures in the microbumps at four representative times during solidification, i.e., $6.3 \times 10^{-8} \mathrm{~s}$, $1.0 \times 10^{-7} \mathrm{~s}, 1.5 \times 10^{-7} \mathrm{~s}$ and $3.0 \times 10^{-7} \mathrm{~s}$, are analyzed in this section. The four times are referred to as Time I to IV hereinafter. The microstructures and the corresponding statistics from wavelet analysis are presented in Figs. 3-6 and Tables I-IV respectively.

At Time I, no substantial difference can be found in the horizontal and vertical detail coefficients for the four microstructures as shown in Table I. The hourglass has a slightly smaller mean horizontal coefficient and a slightly larger mean vertical coefficient. The total energy of the detail coefficients also differs little: $0.058,0.0623,0.0639$ and 0.0652 at level 3 and $0.0038,0.004,0.0042$ and 0.0043 at level 2 for the four geometries, respectively. The dendrites shown in Fig. 3 also exhibit a consistent distribution in both the horizontal and vertical directions.
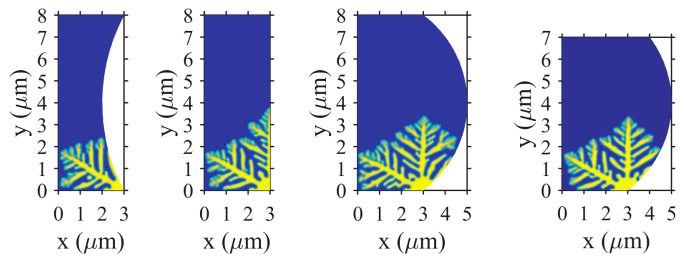

Fig. 3. Microstructure formed in microbumps of four different geometries during solidification at $t=6.3 \times 10^{-8} \mathrm{~s}$.

TABLE I. STATISTICAL DATA ON THE DETAIL COEFFICIENTS AT TIME I FOR MICROBUMPS OF DIFFERENT GEOMETRIES

\begin{tabular}{|c|l|c|c|c|}
\hline \multirow{2}{*}{$\begin{array}{l}\text { Detail } \\
\text { Coeff. }\end{array}$} & Geometry & \multicolumn{3}{|c|}{ Statistics } \\
\cline { 3 - 5 } & & Mean & $\begin{array}{c}\text { Standard } \\
\text { deviation }\end{array}$ & Energy \\
\hline \multirow{4}{*}{ H3 } & Hourglass & 0.0535 & 0.1486 & 0.0249 \\
\cline { 2 - 5 } & Rectangle & 0.0669 & 0.1681 & 0.0327 \\
\cline { 2 - 5 } & Sym. barrel & 0.0692 & 0.1679 & 0.0330 \\
\cline { 2 - 5 } & Asy. barrel & 0.0688 & 0.1705 & 0.0338 \\
\hline \multirow{4}{*}{ V3 } & Hourglass & 0.0672 & 0.1691 & 0.0331 \\
\cline { 2 - 5 } & Rectangle & 0.0653 & 0.1593 & 0.0296 \\
\cline { 2 - 5 } & Sym. barrel & 0.0683 & 0.1621 & 0.0309 \\
\cline { 2 - 5 } & Asy. barrel & 0.0686 & 0.1634 & 0.0314 \\
\hline \multirow{4}{*}{ H2 } & Hourglass & 0.0135 & 0.0382 & 0.0016 \\
\cline { 2 - 5 } & Rectangle & 0.0169 & 0.0429 & 0.0021 \\
\cline { 2 - 5 } & Sym. barrel & 0.0175 & 0.0430 & 0.0022 \\
\cline { 2 - 5 } & Asy. barrel & 0.0173 & 0.0439 & 0.0022 \\
\hline \multirow{5}{*}{ V2 } & Hourglass & 0.0170 & 0.0434 & 0.0022 \\
\cline { 2 - 5 } & Rectangle & 0.0165 & 0.0407 & 0.0019 \\
\cline { 2 - 5 } & Sym. barrel & 0.0173 & 0.0415 & 0.0020 \\
\cline { 2 - 5 } & Asy. barrel & 0.0173 & 0.0421 & 0.0021 \\
\hline
\end{tabular}

At Time II, the energy of the horizontal coefficient for the four geometries are $0.0334,0.0679,0.0681$ and 0.0647 at level 3 and $0.0022,0.0044,0.0045$ and 0.0042 at level 2, respectively. Apart from the hourglass, the other three geometries have a similar horizontal energy being almost the double of the hourglass. However, the four geometries differ little in the vertical energy. This result suggests that the number of dendrites in the horizontal direction is much smaller in hourglass compared to other geometries at Tim II. In other words, there is primarily vertical dendrites growing in the hourglass at time II.

At Time III, the energy of the horizontal coefficient at level 3 for the four geometries are 0.604, 0.0954, 0.1018 and 0.1002, respectively. It is obviously that at this time the energy of the other three geometries is less than the double of the hourglass. 
This suggests that there are dendrites growing in the horizontal direction at a later stage in the hourglass.
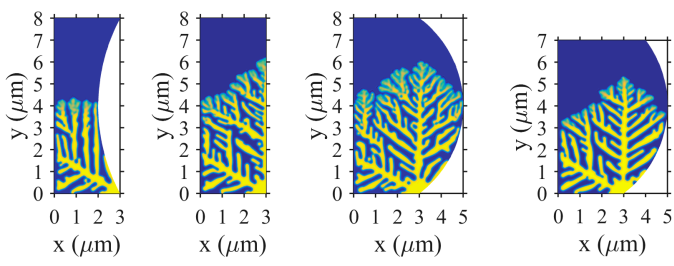

Fig. 4. Microstructure formed in microbumps of four different geometries during solidification at $t=1.0 \times 10^{-7} \mathrm{~s}$.

TABLE II. STATISTICAL DATA ON THE DETAIL COEFFICIENTS AT TIME II FOR MICROBUMPS OF DIFFERENT GEOMETRIES

\begin{tabular}{|l|l|c|c|c|}
\hline \multirow{2}{*}{$\begin{array}{c}\text { Detail } \\
\text { Coeff. }\end{array}$} & Geometry & Statistics \\
\cline { 3 - 5 } & & Mean & $\begin{array}{c}\text { Standard } \\
\text { deviation }\end{array}$ & Energy \\
\hline \multirow{4}{*}{ H3 } & Hourglass & 0.0727 & 0.1675 & 0.0334 \\
\cline { 2 - 5 } & Rectangle & 0.1338 & 0.2237 & 0.0679 \\
\cline { 2 - 5 } & Sym. barrel & 0.1449 & 0.2171 & 0.0681 \\
\cline { 2 - 5 } & Asy. barrel & 0.1270 & 0.2204 & 0.0647 \\
\hline \multirow{4}{*}{ V3 } & Hourglass & 0.0358 & 0.2529 & 0.0842 \\
\cline { 2 - 5 } & Rectangle & 0.1470 & 0.2288 & 0.0740 \\
\cline { 2 - 5 } & Sym. barrel & 0.1689 & 0.2350 & 0.0838 \\
\cline { 2 - 5 } & Asy. barrel & 0.1417 & 0.2290 & 0.0725 \\
\hline \multirow{4}{*}{ H2 } & Hourglass & 0.0183 & 0.0430 & 0.0022 \\
\cline { 2 - 5 } & Rectangle & 0.0338 & 0.0574 & 0.0044 \\
\cline { 2 - 5 } & Sym. barrel & 0.0366 & 0.0558 & 0.0045 \\
\cline { 2 - 5 } & Asy. barrel & 0.0319 & 0.0566 & 0.0042 \\
\hline \multirow{4}{*}{ V2 } & Hourglass & 0.0358 & 0.0647 & 0.0055 \\
\cline { 2 - 5 } & Rectangle & 0.0372 & 0.0586 & 0.0048 \\
\cline { 2 - 5 } & Sym. barrel & 0.0427 & 0.0604 & 0.0055 \\
\cline { 2 - 5 } & Asy. barrel & 0.0356 & 0.0588 & 0.0047 \\
\hline
\end{tabular}
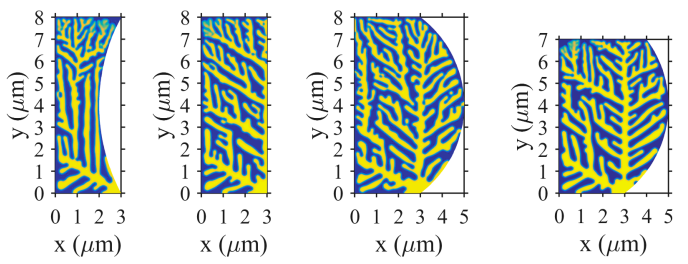

Fig. 5. Microstructure formed in microbumps of four different geometries during solidification at $t=1.5 \times 10^{-7} \mathrm{~s}$.

At Time IV, the total energy of the horizontal and vertical coefficients for the four geometries are $0.1988,0.177,0.1812$ and 0.1876 at level 3 and $0.0128,0.0114,0.0118$ and 0.0122 at level 2, respectively. The total energies of the four geometries become similar at this time. For the hourglass, the vertical energy is almost triple of the horizontal energy at the same level. This indicates that there are primarily vertical dendrites growing in the hourglass at this time. Among the other three geometries, however, the vertical energy is only about $20 \%$ more than the horizontal energy at the same level.

\section{Microstructural Evolution and Wavelet Analysis}

Figs. 7-9 plot the mean, standard deviation and energy of the detail coefficients for the microstructure in microbumps of the four geometries as a function of the time step. It is evident that the microstructural evolution in the hourglass differ from the other three geometries.

TABLE III. STATISTICAL DATA ON THE DETAIL COEFFICIENTS AT TIME III FOR MICROBUMPS OF DIFFERENT GEOMETRIES

\begin{tabular}{|c|l|c|c|c|}
\hline \multirow{2}{*}{$\begin{array}{c}\text { Detail } \\
\text { Coeff. }\end{array}$} & Geometry & Statistics \\
\cline { 3 - 5 } & & Mean & $\begin{array}{c}\text { Standard } \\
\text { deviation }\end{array}$ & Energy \\
\hline \multirow{4}{*}{ H3 } & Hourglass & 0.1367 & 0.2041 & 0.0604 \\
\cline { 2 - 5 } & Rectangle & 0.1882 & 0.2448 & 0.0954 \\
\cline { 2 - 5 } & Sym. barrel & 0.2011 & 0.2477 & 0.1018 \\
\cline { 2 - 5 } & Asy. barrel & 0.1898 & 0.2533 & 0.1002 \\
\hline \multirow{4}{*}{ V3 } & Hourglass & 0.2832 & 0.2987 & 0.1694 \\
\cline { 2 - 5 } & Rectangle & 0.2343 & 0.2697 & 0.1276 \\
\cline { 2 - 5 } & Sym. barrel & 0.2396 & 0.2661 & 0.1282 \\
\cline { 2 - 5 } & Asy. barrel & 0.2293 & 0.2713 & 0.1262 \\
\hline \multirow{4}{*}{ H2 } & Hourglass & 0.0347 & 0.0523 & 0.0039 \\
\cline { 2 - 5 } & Rectangle & 0.0475 & 0.0630 & 0.0062 \\
\cline { 2 - 5 } & Sym. barrel & 0.0507 & 0.0637 & 0.0066 \\
\cline { 2 - 5 } & Asy. barrel & 0.0476 & 0.0651 & 0.0065 \\
\hline \multirow{3}{*}{ V2 } & Hourglass & 0.0712 & 0.0766 & 0.0109 \\
\cline { 2 - 5 } & Rectangle & 0.0590 & 0.0694 & 0.0083 \\
\cline { 2 - 5 } & Sym. barrel & 0.0603 & 0.0684 & 0.0083 \\
\cline { 2 - 5 } & Asy. barrel & 0.0575 & 0.0698 & 0.0082 \\
\hline
\end{tabular}
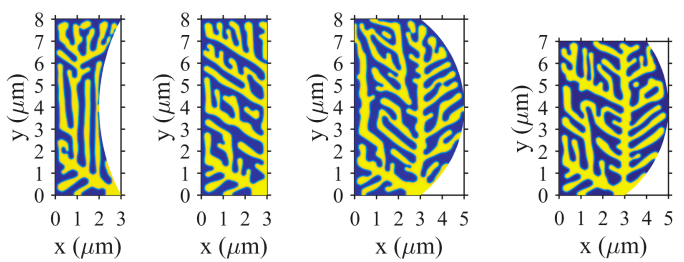

Fig. 6. Microstructure formed in microbumps of four different geometries during solidification at $t=3.0 \times 10^{-7} \mathrm{~s}$.

TABLE IV. STATISTICAL DATA ON THE DETAIL COEFFICIENTS AT TIME IV FOR MICROBUMPS OF DIFFERENT GEOMETRIES

\begin{tabular}{|c|l|c|c|c|}
\hline \multirow{2}{*}{$\begin{array}{l}\text { Detail } \\
\text { Coeff. }\end{array}$} & Geometry & Statistics \\
\cline { 3 - 5 } & & Mean & $\begin{array}{c}\text { Standard } \\
\text { deviation }\end{array}$ & Energy \\
\hline \multirow{4}{*}{ H3 } & Hourglass & 0.0261 & 0.0497 & 0.0484 \\
\cline { 2 - 5 } & Rectangle & 0.1449 & 0.2380 & 0.0777 \\
\cline { 2 - 5 } & Sym. barrel & 0.1571 & 0.2387 & 0.0817 \\
\cline { 2 - 5 } & Asy. barrel & 0.1545 & 0.2456 & 0.0842 \\
\hline \multirow{4}{*}{ V3 } & Hourglass & 0.0603 & 0.0779 & 0.1504 \\
\cline { 2 - 5 } & Rectangle & 0.1792 & 0.2593 & 0.0993 \\
\cline { 2 - 5 } & Sym. barrel & 0.1850 & 0.2554 & 0.0995 \\
\cline { 2 - 5 } & Asy. barrel & 0.1858 & 0.2625 & 0.1034 \\
\hline \multirow{4}{*}{ H2 } & Hourglass & 0.1030 & 0.1943 & 0.0031 \\
\cline { 2 - 5 } & Rectangle & 0.0364 & 0.0610 & 0.0050 \\
\cline { 2 - 5 } & Sym. barrel & 0.0395 & 0.0612 & 0.0053 \\
\cline { 2 - 5 } & Asy. barrel & 0.0386 & 0.0630 & 0.0055 \\
\hline \multirow{4}{*}{ V2 } & Hourglass & 0.2402 & 0.3045 & 0.0097 \\
\cline { 2 - 5 } & Rectangle & 0.0450 & 0.0665 & 0.0064 \\
\cline { 2 - 5 } & Sym. barrel & 0.0465 & 0.0656 & 0.0065 \\
\cline { 2 - 5 } & Asy. barrel & 0.0465 & 0.0673 & 0.0067 \\
\hline
\end{tabular}

Fig. 7 shows that the standard deviations of the detail coefficients level out after the time step 120, which signals the end of the growth stage and the start of the coarsening stage of the dendrites. 


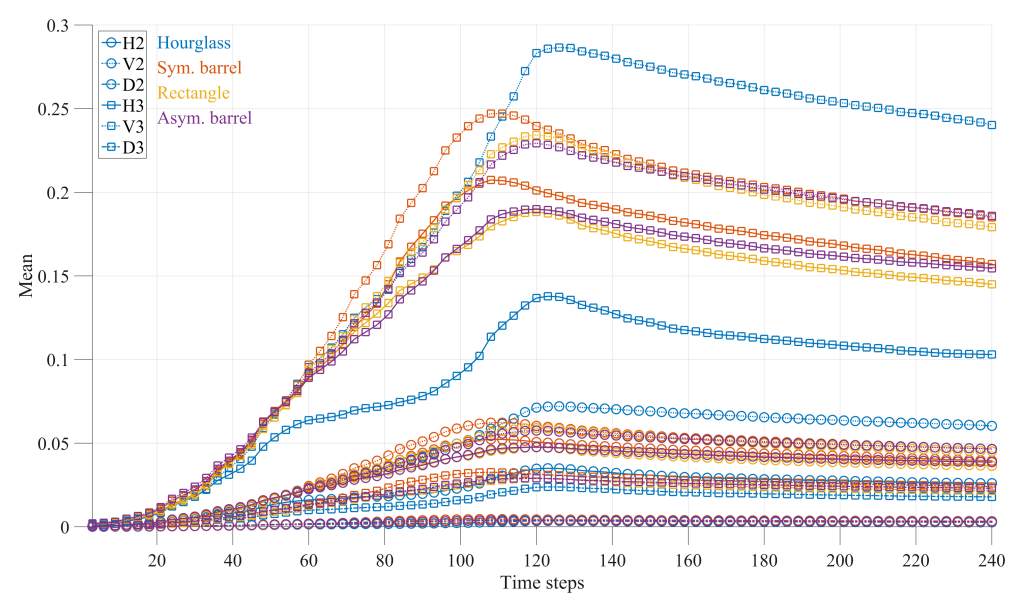

Fig. 7. The mean of the detail coefficents as a function of time step for microbumps of four different geometries.

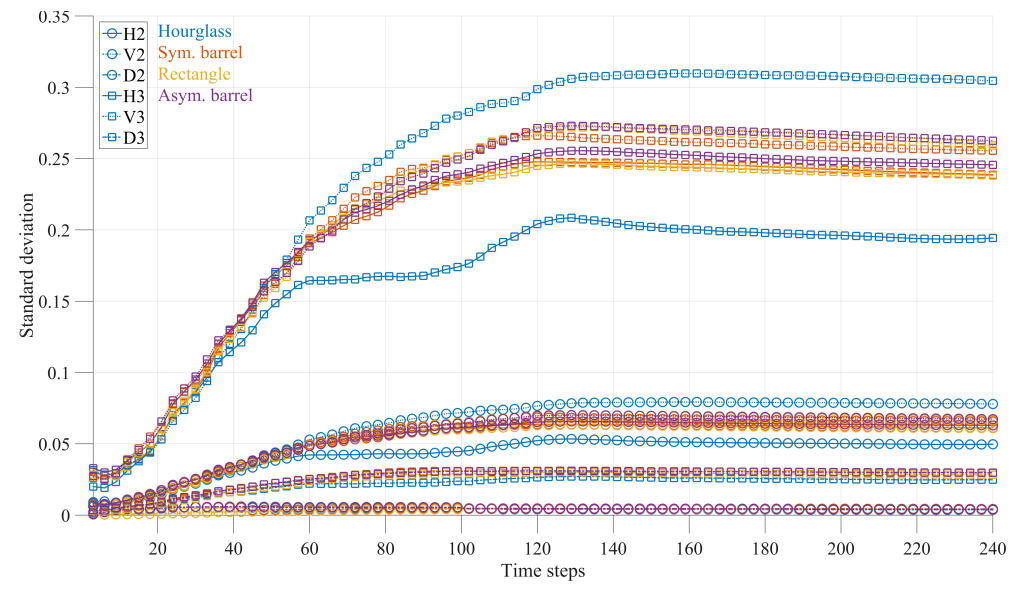

Fig. 8. The standard deviation of the detail coefficents as a function of time step for microbumps of four different geometries.

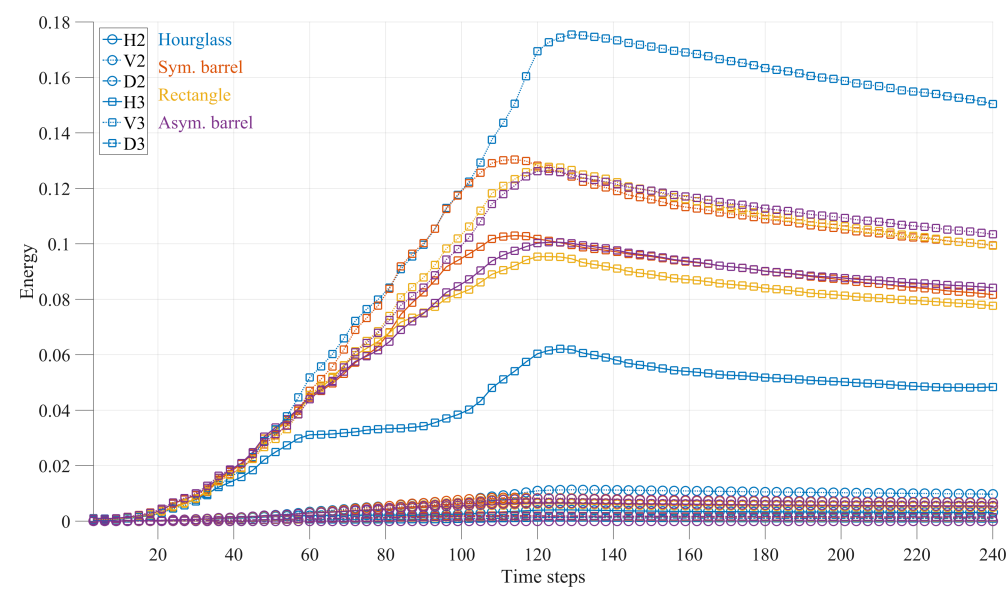

Fig. 9. The energy of the detail coefficents as a function of time step for microbumps of four different geometries. 
The slopes of the energy curves at level 3 can be calculated from Fig. 9 and the result is shown in Table V. From time step 0 to 30 , corresponding to 0 to $3.6 \times 10^{-8} \mathrm{~s}$, the growth rates of the dendrites in the hourglass are similar in both the horizontal and vertical directions. However, the growth rate in the vertical direction doubles that in the horizontal direction from time step 30 to 60 , corresponding to $3.6 \times 10^{-8}$ to $7.5 \times 10^{-8} \mathrm{~s}$. In the other geometries, the dendrites grow with a similar rate in both directions from time step 0 to 60 . This difference in growth behavior between the hourglass and the other three geometries strengthens as time goes on. From time step 60 to 90, corresponding to $7.5 \times 10^{-8}$ to $1.11 \times 10^{-7} \mathrm{~s}$, the dendrites in the hourglass grow in the vertical direction with a speed of 16 times of that in the horizontal direction, which indicates that the dendrites primarily grows in the vertical direction during this stage. In contrast, the dendrites in other geometries grow in the vertical direction only amounts to 1.4 times of that in the horizontal direction during the same time interval. An observation on the microstructure in Fig. 6 can explain the cause for this difference. Compared with the dendrites in the other three geometries, the dendrites in the hourglass miss the 90 primary dendrite. Without any interaction from the 90 primary dendrite in the hourglass, the secondary dendrites branched from the 30 primary dendrite can grow freely in the vertical direction, resulting a substantial increase of vertical dendrites in the microbump. At a later stage, i.e. from time step 90 to 120 , corresponding to $1.11 \times 10^{-7}$ to $1.5 \times 10^{-7} \mathrm{~s}$, the ratio between the vertical and horizontal growth rates becomes 2.67 , $1.94,1.45$ and 1.67 for the four geometries, respectively. There is an increase of this ratio for the latter three geometries. This is due to the fact that the growth front of the dendrites meets the left (and right) boundaries of the microbump during this stage and the dendrites can only develop further in the vertical direction. It is obvious, however, that there is a substantial increase of the growth rate in the horizontal direction in the hourglass at this stage, resulting the ratio decreases to 2.67. Following time step 120 , it can be observed that there is a decrease of energy in both directions due to the microstructural evolution reaching a coarsening stage. During this stage, there is no new dendrite growing but finer and higher order dendrites disappearing, thus resulting a decrease of the detail coefficients. In addition, the rate of the energy decrease is larger in the vertical direction.

\section{Wavelet Analysis on Size Effect}

Wavelet analysis on the microstructural evolution in two sizes of hourglass-shaped microbumps, i.e. $3 \mu \mathrm{m} \times 8 \mu \mathrm{m}$ and 6 $\mu \mathrm{m} \times 16 \mu \mathrm{m}$, is discussed in this section. The simulation time for the $8 \mu \mathrm{m}$ height bump is $3.0 \times 10^{-7} \mathrm{~s}$ with a total of 240 time steps and for the $16 \mu \mathrm{m}$ height bump $5.0 \times 10^{-7} \mathrm{~s}$ and 200 time steps. According to the stages of microstructural evolution, three different times, referred to as Time I to III, are chosen for each size to compare the corresponding microstructure. For the $8 \mu \mathrm{m}$ height bump, $6.3 \times 10^{-8} \mathrm{~s}, 1.5 \times 10^{-7} \mathrm{~s}$ and $3.0 \times 10^{-7} \mathrm{~s}$ are chosen and for the $16 \mu \mathrm{m}$ height bump, $1.05 \times 10^{-7} \mathrm{~s}, 2.5 \times 10^{-7} \mathrm{~s}$ and $5.0 \times 10^{-7} \mathrm{~s}$ are chosen.
TABLE V. SLOPES OF THE HORIZONTAL AND VERTICAL DETAIL COEFFICIENT GROWTH CURVES DURING DIFFERENT STAGES OF SOLIDIFICATION

\begin{tabular}{|c|c|c|c|}
\hline \multirow{2}{*}{ Time step } & \multirow{2}{*}{ Geometry } & \multicolumn{2}{|c|}{$\begin{array}{l}\text { Slope } \\
\end{array}$} \\
\hline & & Horizontal $\left(\times 10^{-4}\right)$ & Vertical $\left(\times 10^{-4}\right)$ \\
\hline \multirow{4}{*}{$0 \sim 30$} & Hourglass & 2.72 & 2.26 \\
\hline & Rectangle & 2.71 & 2.76 \\
\hline & Sym. barrel & 2.62 & 3.18 \\
\hline & Asy. barrel & 3.13 & 3.43 \\
\hline \multirow{4}{*}{$30 \sim 60$} & Hourglass & 7.79 & 15.00 \\
\hline & Rectangle & 12.00 & 12.00 \\
\hline & Sym. barrel & 12.00 & 13.00 \\
\hline & Asy. barrel & 12.00 & 11.00 \\
\hline \multirow{4}{*}{$60 \sim 90$} & Hourglass & 1.04 & 16.00 \\
\hline & Rectangle & 10.00 & 14.00 \\
\hline & Sym. barrel & 13.00 & 18.00 \\
\hline & Asy. barrel & 9.95 & 13.00 \\
\hline \multirow{4}{*}{$90 \sim 120$} & Hourglass & 8.70 & 23.00 \\
\hline & Rectangle & 6.74 & 13.00 \\
\hline & Sym. barrel & 6.44 & 9.31 \\
\hline & Asy. barrel & 8.44 & 14.00 \\
\hline \multirow{4}{*}{$120 \sim 240$} & Hourglass & -1.00 & -1.58 \\
\hline & Rectangle & -1.48 & -2.36 \\
\hline & Sym. barrel & -1.68 & -2.40 \\
\hline & Asy. barrel & -1.34 & -1.90 \\
\hline
\end{tabular}

Fig. 10 shows, from left to right, the snapshots of microstructure in the $8 \mu \mathrm{m}$ and $16 \mu \mathrm{m}$ height bumps at the three chosen times. Table VI to VIII provide the corresponding statistics of the horizontal and vertical detail coefficients from the wavelet analysis on the microstructure at the three times.

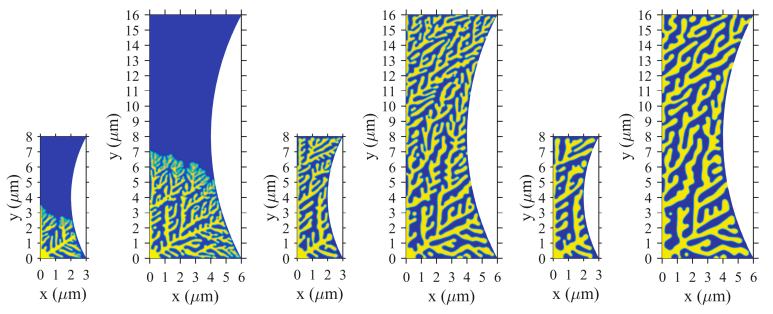

Fig. 10. Microstructure formed in $8 \mu \mathrm{m}$ and $16 \mu \mathrm{m}$ height microbumps at Time I, II and III (from left to right).

Table VI shows that the vertical and horizontal energy at level 3 is slightly larger in the $16 \mu \mathrm{m}$ height bump, which means that the number of dendrites (per unit area) is larger in the larger bump. In addition, the detail coefficients of the larger bump is primarily concentrated at level 4 while the smaller bump level 3 . The result in Table VI suggests, however, there is no major difference in the detail coefficients of the same level in both the vertical and horizontal directions.

It can be observed from Fig. 10 that the growth stage of dendrites completes at Time II in the two sizes of bumps. Table VII shows that at both levels 2 and 3 the energy of the detail coefficient in the smaller bump becomes larger than that in the larger bump at the same level. This result suggests that finer dendrites in the larger bump disappear and thus resulting a decrease in energy in the larger bump. Therefore, the dendrites in the larger bump enter the coarsening stage at Time II. In addition, the vertical coefficient is about $15 \%$ larger than the 
horizontal coefficient at the same level at Time II for both bumps. At Time I, however, the vertical coefficient is only about $6 \%$ larger than the horizontal coefficient. This suggests that the vertical coefficient increases with the microstructure evolution.

The two snapshots on the right side of Fig. 10 show the microstructure at Time III in the two bumps. The dendrites completes the coarsening stage at this time. It is obvious that the energies at different levels in Table VIII are all smaller than the corresponding energy at Time II as shown in Table VII.

TABLE VI. STATISTICAL DATA ON THE DETAIL COEFFICIENTS AT TIME I FOR MICROBUMPS OF DIFFERENT SIZES

\begin{tabular}{|c|c|c|c|c|}
\hline \multirow{2}{*}{$\begin{array}{c}\text { Bump } \\
\text { Height }\end{array}$} & \multirow{2}{*}{$\begin{array}{c}\text { Detail } \\
\text { Coeff. }\end{array}$} & \multicolumn{3}{|c|}{ Statistics } \\
\cline { 2 - 5 } & & Mean & $\begin{array}{c}\text { Standard } \\
\text { deviation }\end{array}$ & Energy \\
\hline \multirow{4}{*}{$8 \mu \mathrm{m}$} & $\mathrm{H} 3$ & 0.0801 & 0.1834 & 0.0400 \\
\cline { 2 - 5 } & $\mathrm{V} 3$ & 0.0884 & 0.1885 & 0.0433 \\
\cline { 2 - 5 } & $\mathrm{H} 2$ & 0.0202 & 0.0469 & 0.0026 \\
\cline { 2 - 5 } & $\mathrm{V} 2$ & 0.0223 & 0.0482 & 0.0028 \\
\hline \multirow{4}{*}{$16 \mu \mathrm{m}$} & $\mathrm{H} 4$ & 0.3562 & 0.7263 & 0.6543 \\
\cline { 2 - 5 } & $\mathrm{V} 4$ & 0.3811 & 0.7442 & 0.6990 \\
\cline { 2 - 5 } & $\mathrm{H} 3$ & 0.0917 & 0.1957 & 0.0467 \\
\cline { 2 - 5 } & $\mathrm{V} 3$ & 0.0987 & 0.2004 & 0.0499 \\
\cline { 2 - 5 } & $\mathrm{H} 2$ & 0.0231 & 0.0501 & 0.0030 \\
\cline { 2 - 5 } & $\mathrm{V} 2$ & 0.0249 & 0.0513 & 0.0033 \\
\hline
\end{tabular}

TABLE VII. STATISTICAL DATA ON THE DETAIL COEFFICIENTS AT TIME II FOR MICROBUMPS OF DIFFERENT SIZES

\begin{tabular}{|c|c|c|c|c|}
\hline \multirow{2}{*}{$\begin{array}{c}\text { Bump } \\
\text { Height }\end{array}$} & \multirow{2}{*}{$\begin{array}{c}\text { Detail } \\
\text { Coeff. }\end{array}$} & \multicolumn{3}{|c|}{ Statistics } \\
\cline { 3 - 5 } & Mean & $\begin{array}{c}\text { Standard } \\
\text { deviation }\end{array}$ & Energy \\
\hline \multirow{3}{*}{$8 \mu \mathrm{m}$} & $\mathrm{H} 3$ & 0.2056 & 0.2542 & 0.1069 \\
\cline { 2 - 5 } & $\mathrm{V} 3$ & 0.2359 & 0.2639 & 0.1253 \\
\cline { 2 - 5 } & $\mathrm{H} 2$ & 0.0517 & 0.0653 & 0.0069 \\
\cline { 2 - 5 } & $\mathrm{V} 2$ & 0.0594 & 0.0679 & 0.0081 \\
\hline \multirow{4}{*}{$16 \mu \mathrm{m}$} & $\mathrm{H} 4$ & 0.7449 & 0.9237 & 1.4080 \\
\cline { 2 - 5 } & $\mathrm{V} 4$ & 0.8483 & 0.9620 & 1.6450 \\
\cline { 2 - 5 } & $\mathrm{H} 3$ & 0.1892 & 0.2525 & 0.0995 \\
\cline { 2 - 5 } & $\mathrm{V} 3$ & 0.2149 & 0.2620 & 0.1148 \\
\cline { 2 - 5 } & $\mathrm{H} 2$ & 0.0477 & 0.0648 & 0.0065 \\
\cline { 2 - 5 } & $\mathrm{V} 2$ & 0.0065 & 0.0674 & 0.0075 \\
\hline
\end{tabular}

TABLE VIII. STATISTICAL DATA ON THE DETAIL COEFFICIENTS AT TIME III FOR MICROBUMPS OF DIFFERENT SIZES

\begin{tabular}{|c|c|c|c|c|}
\hline \multirow{3}{*}{$\begin{array}{c}\text { Bump } \\
\text { Height }\end{array}$} & $\begin{array}{c}\text { Detail } \\
\text { Coeff. }\end{array}$ & \multicolumn{3}{|c|}{ Statistics } \\
\cline { 2 - 5 } & Mean & $\begin{array}{c}\text { Standard } \\
\text { deviation }\end{array}$ & Energy \\
\hline \multirow{4}{*}{$8 \mu \mathrm{m}$} & $\mathrm{H} 3$ & 0.1566 & 0.2443 & 0.0842 \\
\cline { 2 - 5 } & $\mathrm{V} 3$ & 0.1773 & 0.2525 & 0.0952 \\
\cline { 2 - 5 } & $\mathrm{H} 2$ & 0.0952 & 0.0625 & 0.0054 \\
\cline { 2 - 5 } & $\mathrm{V} 2$ & 0.0444 & 0.0647 & 0.0062 \\
\hline \multirow{4}{*}{$16 \mu \mathrm{m}$} & $\mathrm{H} 4$ & 0.5510 & 0.8577 & 1.0391 \\
\cline { 2 - 5 } & $\mathrm{V} 4$ & 0.6494 & 0.9226 & 0.9226 \\
\cline { 2 - 5 } & $\mathrm{H} 3$ & 0.1382 & 0.2315 & 0.0727 \\
\cline { 2 - 5 } & $\mathrm{V} 3$ & 0.1630 & 0.2488 & 0.0884 \\
\cline { 2 - 5 } & $\mathrm{H} 2$ & 0.0347 & 0.0592 & 0.0047 \\
\cline { 2 - 5 } & $\mathrm{V} 2$ & 0.0409 & 0.0637 & 0.0057 \\
\hline
\end{tabular}

Figs. 11 to 13 plot the mean, standard deviation and energy of the detail coefficients at different levels as a function of time step during the microstructural evolution in the two sizes of bumps. The consistency of the data curves for both sizes of bumps, e.g. the shape and trend, in the three figures can be observed. The consistency of detail coefficients from wavelet analysis means that the features of the morphology in the two bumps are consistent. The major difference lies in that the data curves of the larger bump is smoother than that of the smaller bump, indicating a more homogeneous microstructure in the larger bump during the microstructural evolution.

During the early stage of microstructural evolution, i.e. from time step 0 to 60 for the smaller bump and from time step 0 to 40 for the larger bump, the slopes of the energy curve for the smaller bump at level 3 are $8.03 \times 10^{-4}$ and $9.57 \times 10^{-4}$ for the horizontal and vertical directions, respectively. The growth rate in the vertical direction is 1.19 times of that in the horizontal direction. This number is 1.06 for the larger bump at level 4 . This suggests that during the early stage of the microstructural evolution, the growth rates in the horizontal and vertical directions are closer in the larger bump. The reason for this result is because the morphology of the dendrites in the larger bump are relatively more homogeneous and better developed, i.e., existing dendrites of higher orders. During the coarsening stage, for the smaller bump from time step 120 to 240 and for the larger bump from time step 90 to 200 , it is noted that the slopes of the curves are negative. The ratio of the growth rates for the smaller bump at level 3 is 1.32 and for the larger bump at level 4 is 1.05 . The result suggests that the finer dendrites in the vertical direction disappear faster in the smaller bump, while this ratio in the larger bump changes little compared to the same ratio at the growth stage. Therefore, it can be concluded that the dendritic morphology is more homogeneous in the larger bump during the microstructural evolution.

\section{SUMMARY}

This work applies the multi-resolution wavelet analysis to the digital microstructure of solidification in microbumps simulated using a phase field method. For the geometry effect on the solidified microstructure, it has been found that during the first stage of microstructural evolution, the vertical growth rate of the dendrites in the hourglass is two times of the horizontal one. At the same stage, the dendrites in the rectangle, symmetric barrel and asymmetric barrel grow with a similar rate in both the horizontal and vertical directions. During the second stage, the ratio between the growth rates in the vertical and horizontal direction becomes 16 for hourglass and 1.4 for the other three geometries. During the third stage, the ratios of the growth rate are 2.67, 1.94, 1.45 and 1.67 for the four geometries, respectively. During the final coarsening stage, the energy of the detail coefficient decreases in the vertical direction with a rate of $1.58,1.60,1.43$ and 1.42 times of that in the horizontal direction for the four geometries, respectively. For the size effect, it has been found that during the initial stage of the dendritic growth, the vertical growth rate is 1.19 times of the horizontal one in the smaller bump and this number is 1.06 for the larger bump. During the coarsening stage, the energy of detail coefficient decreases in the vertical direction with rate of 1.32 and 1.05 times of that in the horizontal direction for the smaller and larger bump, respectively. 

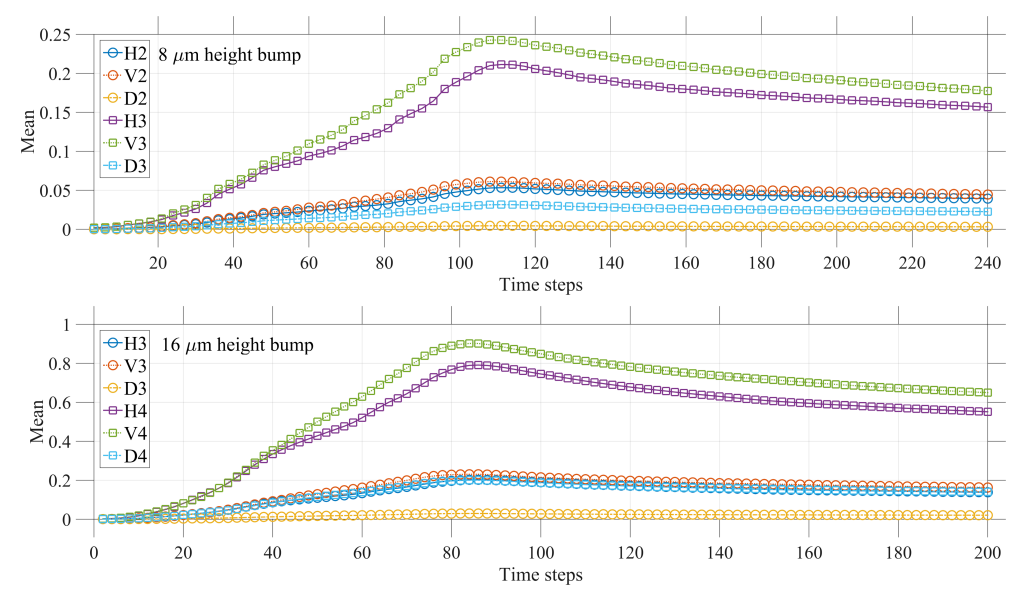

Fig. 11. The mean of the detail coefficents as a function of time step for microbumps of different sizes.
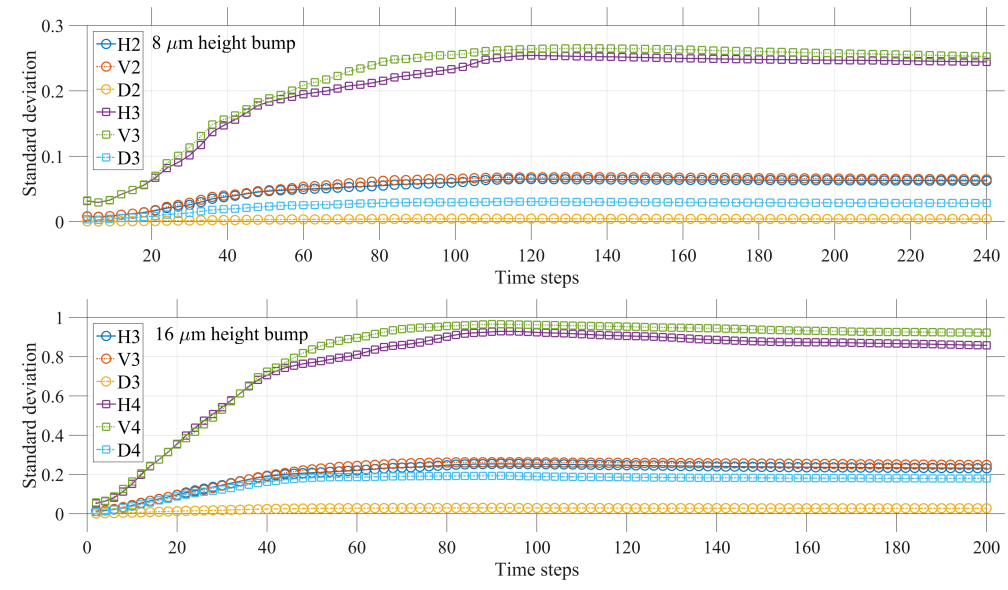

Fig. 12. The standard deviation of the detail coefficents as a function of time step for microbumps of different sizes.
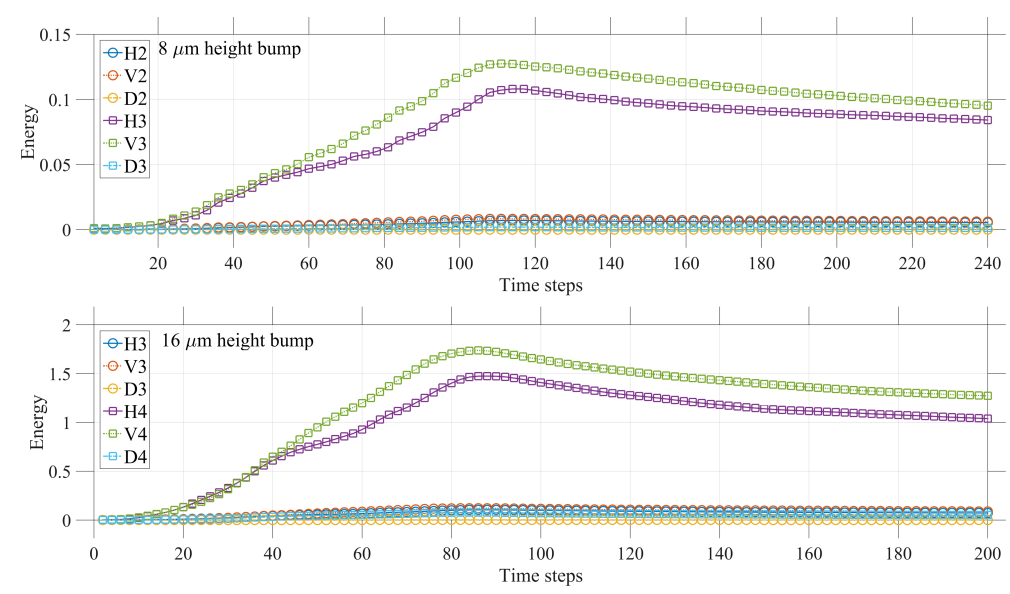

Fig. 13. The energy of the detail coefficents as a function of time step for microbumps of different sizes. 


\section{ACKNOWLEDGMENT}

This work was supported by the Pearl River Science and Technology Nova Program of Guangzhou under Grant 2012J2200074 and the National Natural Science Foundation of China (NSFC) under Grant 51004118. The phase field microstructural modeling on solidification was carried out by Zhiyong Wu during his MSc study under the supervision of Zhiheng Huang at the Sun Yat-sen University.

\section{REFERENCES}

[1] H. Xiong, Z. Huang, D. Wu, Y. Zhang, "The geometrical effects in a model coupled with microstructural evolution and mechanical behavior for small-scale solder joints," Int. Conf. Electronic Packaging Technology \& High Density Packaging (ICEPT-HDP), Guilin, China, 2012, pp. 324-329.

[2] Z. Huang, P.P. Conway, and R.C. Thomson, "Microstructural considerations for ultrafine lead free solder joints," Microelectron. Reliab., vol. 47, pp. 1997-2006, 2007.

[3] Z. Wu, Z. Huang, H. Xiong, P.P. Conway, C. Liu, "Linkages between microstructure and mechanical properties of ultrafine interconnects," J. Electron. Mater., vol. 42, pp. 263-271, 2013.

[4] Z. Wu, "Numerical simulation on microstructural formation in ultrafine interconnects of electroinc packaging," Master Thesis of Sun Yat-sen University, 2013.

[5] S. G. Kim, W. T. Kim, J. S. Lee, M. Ode and T. Suzuki, "Large scale simulation of dendritic growth in pure undercooled melt by phasefield model," ISIJ Int., vol. 39, pp. 335-340, 1999.

[6] COMSOL Multiphysics Reference Manual, Version 5.1, COMSOL, 2015.

[7] D. Lee Fugal, "Conceptual wavelets in digial signal processing," Space \& Signals Techologies LLC, 2009.

[8] Michel Misiti, Yves Misiti, Georges Oppenheim, Jean-Michel Poggi, "Wavelet Toolbox User's Guide Version R2015a", The MathWorks, Inc., 2015. 